\title{
Angiotensin-Converting Enzyme Inhibition Induces Death Receptor Apoptotic Pathways in Erythroid Precursors following Renal Transplantation
}

\author{
Ilya Glezerman ${ }^{a}$ Hiren Patel ${ }^{b}$ Daniel Glicklich ${ }^{a}$ Helena Croizat ${ }^{c}$ \\ Prasad Devarajan ${ }^{b}$
}

Divisions of a Nephrology and ' Hematology, Albert Einstein College of Medicine, Bronx, N.Y., and bephrology, Cincinnati Children's Hospital Medical Center, Cincinnati, Ohio, USA

\section{Key Words \\ Posttransplantation erythrocytosis - Apoptosis · Fas · Angiotensin-converting enzyme inhibition}

\begin{abstract}
Background: Posttransplant erythrocytosis (PTE) is a condition that occurs in kidney transplant patients and is characterized by increase in hematocrit above $51 \%$. While its pathogenesis remains unclear, angiotensinconverting enzyme inhibitors (ACEI) have been used successfully in the treatment of PTE. We have previously shown that ACEI induce apoptosis in the peripheral erythroid precursors from patients with PTE. In the current study we elucidate the molecular mechanisms of ACEIinduced apoptosis. Methods: Peripheral CD34+ cells were obtained from four normal controls, five normal kidney transplants, and six kidney transplants with PTE, before and after treatment with ACEI. We evaluated the expression of a variety of apoptotic factors by quantitative reverse transcription-multiplex polymerase chain reaction, Western blot and immunocytochemistry. $\boldsymbol{R} \boldsymbol{e}$ sults: ACEI resulted in a significant induction of Fas, FADD, and TRADD mRNAs in renal transplant patients with or without PTE. No changes were noted in the
\end{abstract}

\section{KARGER}

Fax +41613061234

E-Mail karger@karger.ch

www.karger.com
(C) 2003 S. Karger AG, Basel

0250-8095/03/0234-0195\$19.50/0

Accessible online at:

www. karger.com/ajn expression of mRNAs encoding $\mathrm{Bcl}-2, \mathrm{Bcl}-\mathrm{xL}$, Bax, caspase 8, caspase 3, or GAPDH. ACEl also resulted in a significant upregulation of Fas, FADD and TRADD protein expression, and their localization predominantly at the plasma membrane. Conclusions: Our results suggest that ACEI therapy induces apoptosis in erythrocyte progenitor cells of renal transplant patients at least in part via induction of death receptor apoptotic cascades.

Copyright @ 2003 S. Karger AG, Basel

\section{Introduction}

Posttransplant erythrocytosis (PTE), defined as an elevation in hematocrit above $51 \%$, is a syndrome unique to renal transplant recipients [1]. It affects between 10 and $15 \%$ of patients in this population, but its pathogenesis remains unclear [1]. Erythropoietin (EPO), angiotensin II and insulin-like growth factor 1 (IGF-1) have been implicated in PTE [2-7], and angiotensin-converting enzyme inhibitors (ACEI) have been successfully used in the treatment [1]. Although there is evidence that ACEI may cause anemia by reducing EPO levels in renal transplant recipients [8], we have previously documented an EPO-independent inhibition of cell growth by ACEI in erythroid
Prasad Devarajan, MD

Division of Nephrology and Hypertension, Cincinnati Children's Hospital Medical Center 3333 Burnet Avenue, MLC 7022

Cincinnati, OH 45229-3039 (USA)

Tel. +1 513636 4531, Fax +1 513636 7407, E-Mail prasad.devarajan@cchmc.org 
progenitor cells from patients with PTE [4]. Since ACEI have been shown to have a profound effect on programmed cell death in various tissues [9-14], we examined in a previous study the effects of ACEI on erythroid precursors in patients with PTE, and demonstrated a significant increase in apoptosis rate [15]. The underlying molecular mechanisms, however, remain unclear.

Apoptosis is a complex process of programmed cell death triggered either by cessation of signals for cell survival or arrival of signals promoting cell demise. In recent years, specific proteases belonging to the caspase family have surfaced as crucial effectors of apoptosis, and the major intracellular apoptotic pathways may be classified according to the type of pro-caspase that is activated. Activation of the initiator pro-caspase 8 results from signaling via plasma membrane death receptors such as Fas and signal transducing ligands such as FADD (Fas-associating protein with death domain) and TRADD (TNF receptor-associating protein with death domain) [16]. On the other hand, activation of the initiator pro-caspase 9 is dependent primarily on mitochondrial signaling pathways regulated by members of the Bcl-2 family [17]. Activation of pro-apoptotic Bcl-2 family members such as Bax can trigger a sequence of events leading to release of mitochondrial cytochrome $\mathrm{c}$ into the cytosol, and activation of pro-caspase 9 . Once activated, both caspases 8 and 9 participate in a cascade that culminates in the activation of caspase 3, which cleaves several substrates resulting in chromosomal DNA fragmentation and cellular morphologic changes characteristic of apoptosis [18]. Anti-apoptotic members of the Bcl-2 family such as Bcl-2 and Bcl$\mathrm{xL}$ can play a pivotal protective role by inhibiting mitochondrial cytochrome c release [19].

In our current investigation, we have attempted to characterize the pathways involved in the ACEI-induced apoptosis of erythroid precursor cells from PTE patients. Our results suggest that this occurs at least in part via activation of cell surface death receptor cascades.

\section{Materials and Methods}

\section{Patients}

Samples for this study were obtained from 6 renal transplant patients diagnosed with PTE. Samples were also obtained from 5 control renal transplant patients with normal hematocrit, and from an additional 4 normal non-transplant controls. All transplant patients had stable renal function and were 6 months or more posttransplantation. PTE was defined as hematocrit of $51 \%$ or greater on at least three consecutive measurements and an increased red cell mass as determined by 51-chromium-labeled red cells. Other causes of erythrocytosis were excluded based on clinical and laboratory testing, as described previously $[4,15]$. The transplant patients without PTE were matched with PTE patients for gender, age, serum creatinine, transplant duration, and maintenance immunosuppression. The detailed demographics of these patients have been previously reported [15]. None of the subjects were on ACEI or AT1 blocker therapy at the beginning of the study. All subjects were treated with lisinopril or fosinopril $(9.25 \pm 1.8 \mathrm{mg} /$ day; range $5-10 \mathrm{mg} /$ day $)$ for the mean duration of $6.8 \pm 4.6$ weeks. The treatment end-point for PTE patients was a reduction in hematocrit back to baseline levels, and transplant patients without PTE were treated for a similar duration. Blood samples were obtained prior to the initiation and immediately upon the completion of the course of treatment [15]. The study protocol was approved by the Institutional Review Board of Montefiore Medical Center, and all subjects gave written informed consent before entering the study.

\section{Isolation of Erythroid Precursors}

Circulating erythrocyte progenitor cells were isolated as previously described $[4,15,20]$. Briefly, $80 \mathrm{ml}$ of blood was diluted $1: 1$ with serum-free a-minimal essential medium, and the low-density mononuclear cells isolated by centrifugation over Ficoll-Hypaque. The lineage-negative $\mathrm{CD} 34+$ cells were isolated as recommended by the manufacturer (Stem Cell Technologies, Vancouver, BC), and stored at $-70^{\circ} \mathrm{C}$. Equal aliquots of each sample were used for documentation of apoptosis by annexin assay in a double blind fashion as previously described [15]. Equal aliquots were also subjected to reverse transcription-polymerase chain reaction, Western analysis, and immunocytochemistry as detailed below, in a double-blind fashion.

\section{Quantitative Reverse Transcription-Multiplex Polymerase Chain}

\section{Reaction (QRT-MPCR)}

This technique was used as an initial screen for identification of apoptotic factors whose expression may be altered in erythroid precursor cells of PTE patients following ACEI. QRT-MPCR is an adaptation of the PCR technique in which various targets are amplified simultaneously in the same reaction by using multiple primer pairs targeted to different gene sets. Differences in expression of target genes may be determined by normalizing their expression against a housekeeping gene such as GAPDH. Briefly, RNA was extracted from erythroid precursor cells using the RNeasy kit (Qiagen, Valencia, Calif., USA), and quantitated by spectrophotometry. Reverse transcription was performed using the Titan One Tube kit (Roche Diagnostics, Indianapolis, Ind., USA). For this study, QRT-MPCR was performed using the Human Apoptosis Gene Sets 2 and 4 according to the manufacturer's instructions (Biosource International, Camarillo, Calif., USA). Genes amplified using primers in set 2 include GAPDH and members of the mitochondrial apoptotic pathways (Bcl-xL, caspase 3, Bax, and Bcl-2). Primers in set 4 target GAPDH and genes in the death receptor pathways (caspase 8, Fas, Fas ligand, FADD, and TRADD). PCR products were analyzed by $2 \%$ agarose gel electrophoresis followed by ethidium bromide staining. Gel bands were scanned and quantitated by densitometry. The relative expression of each apoptotic gene was determined by normalizing its expression against that of GAPDH. The results are expressed as fold changes in gene expression relative to GAPDH.

\section{Western Analysis}

Protein concentrations of cell lysates were determined by the Bradford assay and $10 \mu \mathrm{g}$ of total protein was loaded in each lane. Samples were subjected to SDS-PAGE and transferred to nitrocellu- 
Table 1. Effects of ACEI therapy

\begin{tabular}{|c|c|c|c|c|c|c|}
\hline & \multicolumn{2}{|c|}{ Transplant PTE $(n=6)$} & \multicolumn{2}{|c|}{ Transplant controls $(n=5)$} & \multicolumn{2}{|c|}{ Normal controls $(n=4)$} \\
\hline & pre & post & pre & post & pre & post \\
\hline \multirow{2}{*}{$\begin{array}{l}\text { НCT, \% } \\
\text { Apop, \% }\end{array}$} & $52.5 \pm 1.1$ & $44.3 \pm 2.6^{\mathrm{a}}$ & $43.2 \pm 3.3^{b}$ & $40.8 \pm 2.6^{\mathrm{a}, \mathrm{b}}$ & $45.0 \pm 2.4^{\mathrm{c}}$ & $42.5 \pm 2.5^{\mathrm{a}, \mathrm{c}}$ \\
\hline & $8.3 \pm 6.1$ & $58.3 \pm 12.9^{\mathrm{a}}$ & $11.0 \pm 5.5$ & $63.0 \pm 13.9^{\mathrm{a}}$ & $9.3 \pm 3.0$ & $8.3 \pm 3.5^{\mathrm{c}}$ \\
\hline \multicolumn{7}{|c|}{$\begin{array}{ll}\text { a } & p<0.05 \text { pre vs. post ACEI. } \\
\text { b } & p<0.05 \text { transplant controls vs. PTE. } \\
\text { c } & p<0.05 \text { normal controls vs. PTE. }\end{array}$} \\
\hline
\end{tabular}

lose paper. After blocking with $5 \%$ milk for $60 \mathrm{~min}$ at room temperature, primary antibodies were applied for $60 \mathrm{~min}$ at room temperature as recommended by the manufacturers. Monoclonal antibodies to Fas, FADD and TRADD (Transduction Laboratories, Lexington, Ky., USA) were employed at 1:2,500, 1:200, and 1:500 dilutions, respectively. Following incubation with HRP-conjugated secondary antibodies, immunodetection of transferred proteins was performed using enhanced chemiluminescence (Amersham, Arlington Heights, Ill., USA). Protein bands were quantitated by densitometry.

\section{Immunocytochemistry}

Cells (20 $\mu$ in suspension) were spread on glass slides, air-dried, fixed with $4 \%$ formaldehyde/PBS for $25 \mathrm{~min}$ at $4{ }^{\circ} \mathrm{C}$, permeabilized with $0.2 \%$ Triton X/PBS for $5 \mathrm{~min}$ on ice and blocked with goat serum for $1 \mathrm{~h}$ at room temperature. Monoclonal antibodies to Fas, FADD and TRADD were applied at dilutions recommended by the manufacturer in $2 \% \mathrm{BSA} / 10 \%$ goat serum for $1 \mathrm{~h}$ at room temperature. After washing samples with PBS, flourescein-conjugated goat anti-mouse (GAM-Cy2) secondary antibody was added at 1:2,000 dilution in $0.5 \% \mathrm{BSA} / \mathrm{PBS}$ for $1 \mathrm{~h}$ at room temperature in the dark. Unbound antibodies were removed by gentle washing with PBS. Cells were mounted with Crystal-Mount (Foster City, Calif., USA), and visualized with a microscope (Olympus AX70, Lake Success, N.Y., USA) equipped for epilumination.

\section{Statistics}

All data are expressed as mean \pm SD. The unpaired t test was used to compare means between two groups, and the paired t test to compare data within the same group. ANOVA was used to compare results among three groups. Univariate correlations were evaluated by the Spearman test. All experimental values were obtained by densitometry. The relative expressions of Fas, FADD, and TRADD (mRNA and protein) were compared within each patient before and after ACEI using the paired $t$ test. $\mathrm{p}<0.05$ was considered significant.

\section{Results}

\section{ACEI Induces Apoptosis of Erythroid Precursors in Renal Transplant Patients}

We evaluated the effects of ACEI therapy on clinical parameters and erythroid precursors in 6 renal transplant patients with PTE, 5 matched renal transplant patients without PTE, and 4 normal controls. The demographics of these subjects have been previously reported [15]. The salient clinical and laboratory effects of ACEI therapy are shown in table 1. As expected, the baseline hematocrit of patients with PTE was markedly higher than in both control groups. ACEI therapy resulted in a significant reduction in hematocrit in all three groups (table 1). There was a $15.7 \%$ reduction in average hematocrit in patients with PTE, whereas transplant controls and non-transplant controls displayed a much smaller degree of change in hematocrit $(5.6 \%$ reduction in average hematocrit in both these control groups). Baseline apoptosis rates of erythroid precursor cells were similar in all three groups. Following ACEI therapy, there was a significant and comparable increase in the number of apoptotic erythroid precursors in renal transplant patients, with or without PTE, whereas ACEI did not change the apoptosis rate in normal controls.

\section{ACEI-Induced Apoptosis of Erythroid Precursors Is Associated with Increased Expression of Fas, FADD and TRADD mRNAs}

Since ACEI results in apoptotic cell death of erythroid precursors in renal transplant recipients, both with and without PTE, we employed the quantitative reverse transcription-multiplex polymerase chain reaction (QRTMPCR) to screen for apoptotic factors whose expression may be altered in erythroid precursor cells following 


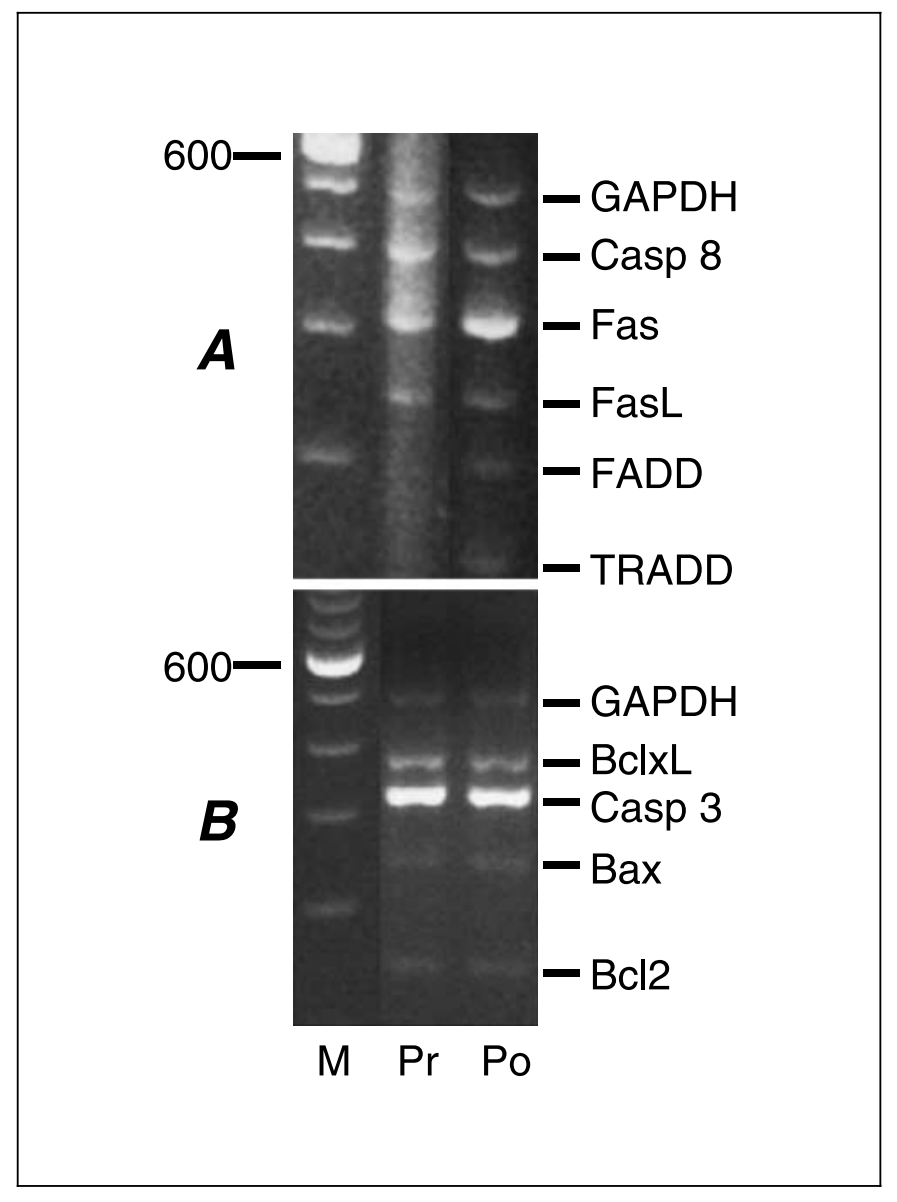

Fig. 1. Representative QRT-MPCR of RNA extracted from erythroid precursors of a subject with PTE, with primers targeted to the genes denoted on the right. A The death receptor pathway. B The mitochondrial pathway. $\mathrm{M}=100 \mathrm{bp}$ marker; $\mathrm{Pr}=$ pre-ACEI; $\mathrm{Po}=$ post-ACEI. ACEI-induced apoptosis of erythroid precursors is associated with increased expression of Fas, FADD and TRADD mRNAs.

ACEI. We consistently detected a significant upregulation of mRNAs encoding Fas, FADD, and TRADD in all renal transplant patients examined, both with and without PTE (fig. 1). No changes were noted in the relative expression of mRNAs for Bcl-2, Bcl-xL, Bax, caspase 8, caspase 3, or GAPDH. Densitometric analysis revealed a relative fold increase of $3 \pm 0.5$ for Fas, $2.6 \pm 0.6$ for FADD, and $2.1 \pm 0.8$ for TRADD expression $(\mathrm{p}<0.05$ in all 3 cases; fig. 2). These changes were quantitatively similar in renal transplant patients, both with and without PTE. No changes were noted in mRNA expression for Fas, FADD, or TRADD in erythroid precursors of normal individuals following ACEI.
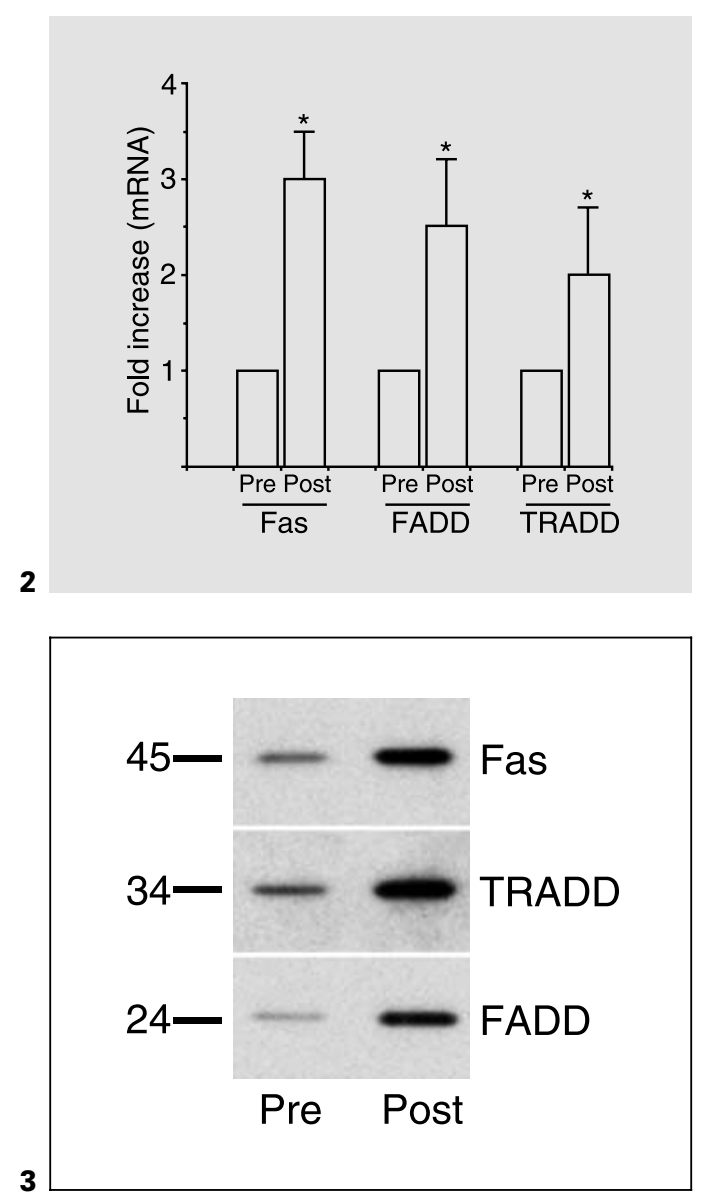

Fig. 2. Densitometric analysis revealed a relative fold increase of $3 \pm 0.5$ for Fas, $2.6 \pm 0.6$ for FADD, and $2.1 \pm 0.8$ for TRADD mRNA expression. Figures are means \pm SD of 6 patients examined. Pre $=$ Pre-ACEI; Post $=$ post-ACEI. ${ }^{*}$ p $<0.05$.

Fig. 3. Representative Western analysis of proteins from erythroid precursors of a subject with PTE, with antibodies to the proteins denoted on the right. Molecular weights (in $\mathrm{kD}$ ) are on the left. Pre = Pre-ACEI; Post = post-ACEI. ACEI-induced apoptosis of erythroid precursors is associated with increased expression of Fas, FADD and TRADD proteins.

\section{ACEI-Induced Apoptosis of Erythroid Precursors Is Associated with Increased Expression of Fas, FADD and TRADD Protein}

We used Western analysis to detect changes in protein expression in erythroid precursor cells of PTE patients following ACEI. We detected a significant upregulation of Fas, FADD, and TRADD protein in all patients examined (fig. 3). Densitometric analysis revealed a fold increase of $4.1 \pm 0.6$ for Fas, $3.6 \pm 0.8$ for FADD, and 3.6 \pm 0.9 for TRADD expression following ACEI $(\mathrm{p}<0.05$ in all 3 cases; fig. 4). No changes were noted in expression of 


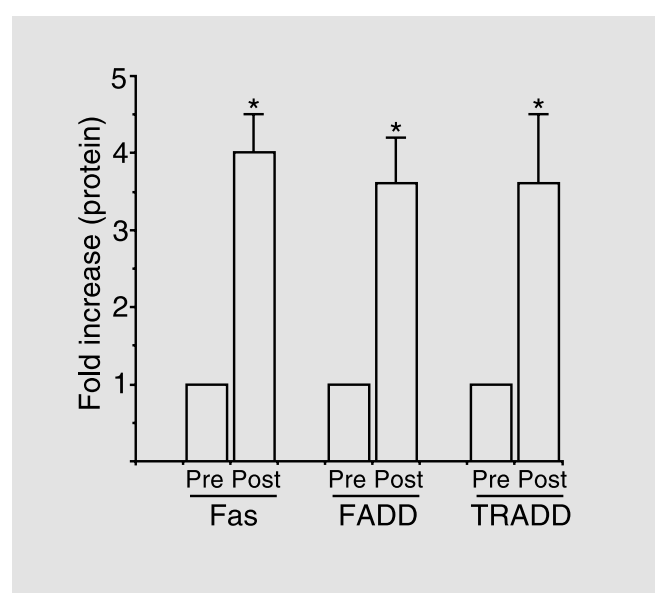

Fig. 4. Densitometric analysis revealed a fold increase of $4.1 \pm 0.6$ for Fas, $3.6 \pm 0.8$ for FADD, and $3.6 \pm 0.9$ for TRADD protein expression. Figures are means $\pm \mathrm{SD}$ of 6 patients examined. Pre $=$ Pre-ACEI, Post $=$ post-ACEI. $*$ p $<0.05$.

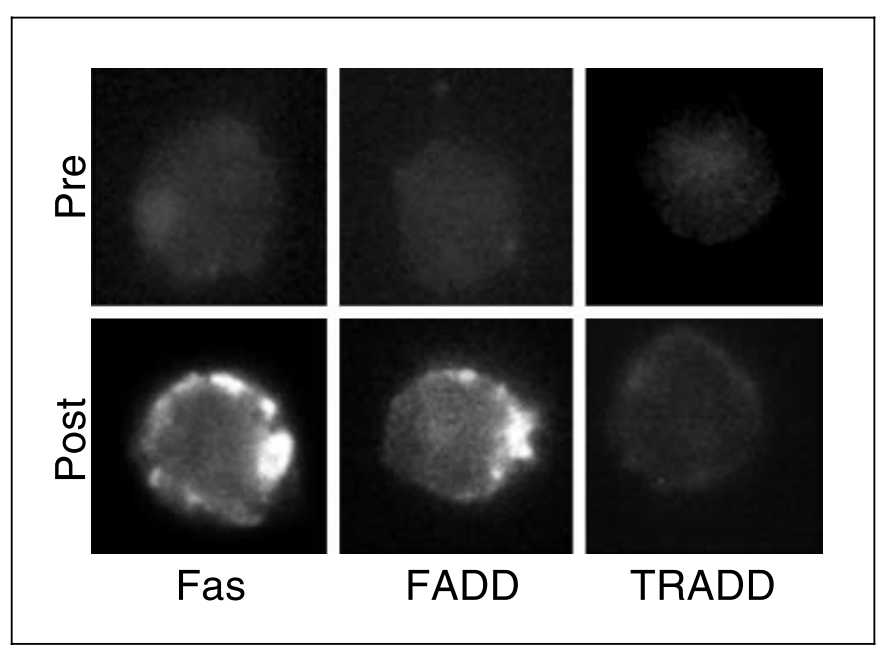

Fig. 5. Representative immunocytochemical analysis of Fas, FADD, and TRADD protein expression in erythroid precursors of a subject with PTE. Pre = Pre-ACEI; Post = post-ACEI .

Fas, FADD, or TRADD protein in erythroid precursors of normal individuals following ACEI.

These results were confirmed by immunocytochemistry. Prior to ACEI therapy, erythroid precursor cells showed only minimal staining with Fas, FADD, or TRADD antibodies, predominantly in a diffuse cytosolic pattern (fig. 5, top panel). Following ACEI, we detected a marked increase in immunostaining for Fas and FADD, and a less impressive change in TRADD expression (fig. 5, bottom panel). Furthermore, Fas and FADD (and to a lesser degree TRADD) now assumed a predominantly plasma membrane localization, with concentrations at sites of plasma membrane blebbing.

\section{Discussion}

PTE remains an enigmatic syndrome unique to renal transplant recipients, in whom the condition can lead to significant morbidity [1]. The therapeutic benefits of ACEI have been attributed at least in part to induction of erythroid precursor cell apoptosis [15], but the underlying molecular mechanisms are unknown. In the present study, following ACEI therapy, we noted a significantly greater reduction in the hematocrit of patients with PTE ( $15.6 \%$ change) when compared to that in non-PTE transplant controls or non-transplant controls $(5.6 \%$ change in both these groups). However, transplant patients both with and without PTE exhibited a dramatic increase in the number of apoptotic erythrocyte precursor cells, when compared to non-transplanted controls. Thus, the ACEImediated increase in progenitor cell apoptosis may explain only in part the measured ACEI-related decrease in hematocrit in patients with PTE. It is also likely that in the complex posttransplantation milieu, other factors such as upregulation of receptors, changes in cytokine environment, immunosuppressive medications, and alterations in cell cycling interact with ACEI therapy to produce apoptosis of erythroid progenitor cells in both PTE and non-PTE patients. Furthermore, it is possible that some other critical event(s) may render the erythrocytes in PTE patients more susceptible to the ACEI therapy, when compared to transplanted patients without PTE.

In this study, we have detected a significant upregulation of mRNAs encoding Fas and FADD, and their respective protein products, suggesting that the apoptosis occurs via the cell surface receptor-activated cascade. This change occurred in both PTE and non-PTE patients following ACEI therapy. In this pathway, an apoptotic stimulus induces the oligomerization of 'death' receptors such as Fas at the cell surface, with resultant recruitment of intracellular signal transduction molecules such as FADD and activation of caspases [16]. Our finding of localization and clustering of Fas and FADD at sites of apoptotic plasma membrane blebbing lend further support to the notion that they play a role in the programmed cell death of erythroid precursors following ACEI. This is a novel and unexpected observation, since angiotensin II has recently been implicated in the initiation of the Fas 
apoptotic cascade [21], and indeed ACEI have been shown to inhibit Fas-mediated cell death in the human lung epithelium and human activated peripheral $\mathrm{T}$ cells $[14,22]$. It is worth noting that in spite of the demonstrated activation of death receptor pathways, we did not detect a significant change in mRNAs encoding either caspase 8 or caspase 3 following ACEI therapy. This may be explained by the fact that caspases normally exist in resting cells as inactive pro-caspases, and induce apoptosis not by increased mRNA transcription but predominantly via post-translational modification, namely by cleavage of the pre-existing pro-enzyme in response to activation of signal transduction pathways [18].

Another putative pro-apoptotic factor that was significantly expressed in erythroid precursors following ACEI was TRADD. These data are more difficult to interpret, since TRADD-dependent downstream signaling mechanisms are more complex, and comprise of both pro-apoptotic and anti-apoptotic pathways [16]. Indeed, emerging evidence points towards a major role for TRADD as a survival factor, and signaling through the TNFR-1/ TRADD cascade rarely induces apoptosis unless protein synthesis is simultaneously inhibited [16]. The specific role of TRADD in PTE is unclear at this time.

A limitation of our study is that we have not exhaustively examined the myriad of apoptotic pathways that may be involved in ACEI-induced apoptosis in PTE. For example, we have not explored the role of TNFR-1 and TRAIL, two related death receptor-dependent pathways [16]. Another limitation is that overexpression and membrane localization of Fas and FADD, albeit highly suggestive, do not necessarily implicate these factors in the ensuing cell death. Confirmation of their role must await future studies documenting the specific activation of caspase 8 , and perhaps in vitro experiments examining the role of inhibitors such as Fas peptides and monoclonal antibodies [23].

As a corollary, our observations do offer a possible mechanism for the development of PTE. We have consistently noted that prior to ACEI, erythroid precursors in patients with PTE display a low expression of Fas mRNA, and minimal expression of FADD mRNA (fig. 1). Furthermore, the expression of Fas and FADD protein is barely detectable by Western blot (fig. 3) and immunocytochemistry (fig. 5), and localized in a diffuse cytosolic pattern. These observations suggest that PTE may represent a condition characterized by downregulation of 'physiologic' apoptotic pathways in erythroid precursors, with resultant erythrocytosis. We recognize, however, that erythropoiesis represents a complex interplay of multiple growth factors, cytokines, and growth inhibitors [24], and it will be important in future studies to dissect out the roles of these various factors in the pathogenesis of PTE.

\section{References}

1 Gaston R, Julian B, Curtis J: Posttransplant erythrocytosis: An enigma revisited. Am J Kid Dis $1994 ; 24: 1-11$.

2 Dagher FJ, Ramos E, Erslev AJ, Alongu SV, Karmi SA, Caro J: Are the native kidneys responsible for erythrocytosis in renal allorecipient? Transplantation 1979;28:496-498.

3 Aeberhard JM, Schneider PA, Vallotton MB, Kurtz A, Leski M: Multiple site estimates of erythropoietin and renin in polycythemic kidney transplant patients. Transplantation 1990; 50:613-616.

4 Glicklich D, Kapoian T, Mian H, Gilman J, Tellis V, Croizat H: Effects of erythropoetin, angiotensin II, and angiotensin-converting enzyme inhibitor on erythroid precursors in patients with posttransplantation erythrocytosis. Transplantation 1999;68:62-66.

5 Morrone LF, Di Paolo S, Logoluso F, Schena A, Stallone G, Giorgino F, Schena FP: Interference of angiotensin-converting enzyme inhibitors on erythropoiesis in kidney transplant recipients. Transplantation 1997;64:913-918.
6 Navarro JF, Garcia J, Macia M, Mora C, Chahin J, Gallego E, Mendez ML, del Castillo N: Effects of losartan on the treatment of posttransplant erythrocytosis. Clin Nephrol 1998; 49:370-372.

7 Julian BA, Brantley RR, Barker CU, Stopka T, Gaston RS, Cutris JJ, Lee JY, Prchal JT: Losartan, an angiotensin II type 1 receptor antagonist, lowers hematocrit in posttransplant erythrocytosis. J Am Soc Nephrol 1998;9:11041108.

8 Gossmann J, Thurmann P, Bachmann T, Weller S, Kachel HG, Schoeppe W, Scheuermann $\mathrm{EH}$ : Mechanism of angiotensin converting enzyme inhibitor-related anemia in renal transplant recipients. Kidney Int 1996;50:973-978.

9 Sharifi AM, Schiffrin EL: Apoptosis in vasculature of spontaneously hypertensive rats: Effect of angiotensin converting enzyme inhibitor and calcium channel antagonist. Am J Hypertens 1998;11:1108-1116.

10 Holm AM, Andersen CB, Haunso S, Hansen PR: ACE-inhibition promotes apoptosis after balloon injury of rat carotid arteries. Cardiovasc Res 2000;45:777-782.
11 Wang LX, Ideishi M, Yahiro E, Urata H, Arakawa K, Saku K: Mechanism of cardioprotective effect of inhibition of the renin-angiotensin system on ischemia/reperfusion-induced injury. Hypertens Res 2001;24:179-187.

12 Goussev A, Sharov VG, Shimoyama H, Tanimura M, Lesch M, Goldstein S: Effects of ACE inhibition on cardiomyocyte apoptosis in dogs with heart failure. Am J Physiol 1998;275: H626-H631.

13 Kelly DJ, Tolcos M, Cooper ME, WilkinsonBerka JL, Gilbert RE: Attenuation of tubular apoptosis by blockade of renin-angiotensin system in diabetic Ren-2 rats. Kidney Int 2002;61: 31-39.

14 Uhal BD, Gidea C, Bargout R, Bifero A, Ibarra-Sunga O, Papp M, Flynn K: Captopril inhibits apoptosis in human lung epithelial cells: A potential antifibrotic mechanism. Am J Physiol 1998;275:L1013-L1017. 
15 Glicklich D, Burris L, Urban A, Tellis V, Goldstein S, Schechner R, Devarajan P, Croizat H: Angiotensin-converting enzyme inhibition induces apoptosis in erythroid precursors and affects insulin-like growth factor- 1 in posttransplantation erythrocytosis. J Am Soc Nephrol 2001;12:1958-1964.

16 Ashkenazi A, Dixit VM: Death receptors: Signaling and modulation. Science 1998;281: 1305-1308.

17 Adams JM, Cory S: The Bcl-2 protein family: arbiters of cell death. Science 1998;281:13221326.

18 Thornberry NA, Lazebnik Y: Caspases: Enemies within. Science 1998;281:1312-1316.
19 Saikumar P, Dong W, Mikhailov V, Denton M, Weinberg JM, Venkatachalam MA: Apoptosis: Definition, mechanisms, and relevance to disease. Am J Med 1999;107:489-506.

20 Croizat H, Billet H, Nagel R: Heterogeneities in the properties of burst forming units of erythroid lineage in sickle cell anemia. Blood 1990;75:1006-1010.

21 Wang R, Zagariya A, Ibarra-Sunga O, Gidea C, And E, Deshmukh S, Chaudhary G, Baraboutis J, Filippatos G, Uhal BD: Angiotensin II induces apoptosis in human and rat alveolar epithelial cells. Am J Physiol 1999;276:L885L889.
22 Odaka C, Mizuochi T: Angiotensin-converting enzyme inhibitor captopril prevents activation-induced apoptosis by interfering with $\mathrm{T}$ cell activation signals. Clin Exp Immunol 2000; 121:515-519.

23 Dhein J, Walczak H, Baumler C, Debatin K-M, Krammer PH: Autocrine T-cell suicide mediated by APO1/Fas/CD95. Nature 1995;373: 438-441.

24 Papayannopoulou T, Abkowitz J: Biology of erythropoiesis, erythroid differentiation and maturation; in Hoffman R, Benz EJ, Shattil SJ, Fune B, Cohen HJ, Silberstein LE (eds): Hematology - Basic Principles and Practice. New York, Churchill-Livingstone, 1995, pp 242254. 